PROCEEDINGS OF THE

AMERICAN MATHEMATICAL SOCIETY

Volume 126, Number 12, December 1998, Pages 3733-3741

S $0002-9939(98) 04461-X$

\title{
ABSOLUTE FIXED POINT SETS FOR MULTI-VALUED MAPS
}

\author{
ERIC L. MCDOWELL
}

(Communicated by Alan Dow)

\begin{abstract}
The notion of a multi-valued absolute fixed point set (MAFS) will be defined and characterized in the setting of set-valued maps with images containing multiple components.
\end{abstract}

\section{INTRODUCTION}

The notion of an absolute fixed point set, in the setting of single-valued maps, was first studied in [1], [2], and [3]; in [3], Martin characterized the class of finite dimensional absolute fixed point sets as the class of finite dimensional absolute retracts. The notion of an absolute fixed point set was extended in [4] to the context of continuum-valued maps and a completely inherent characterization for the notion was obtained. In this paper, we relax the requirement that the maps be continuum-valued and we obtain a characterization for the resulting notion.

A compactum is a nonempty compact metric space, and a continuum is a connected compactum. If $Z$ is a compactum, then $C(Z)$ denotes the space of all subcontinua of $Z$ with the Hausdorff metric and $2^{Z}$ denotes the space of all subcompacta of $Z$ with the Hausdorff metric [5]. A map is a continuous function, and a map into some $2^{Z}$ will be referred to as a multi-valued map; in particular, a map into some $C(Z)$ will be said to be continuum-valued.

A fixed point of a multi-valued map $F$ is a point, $p$, such that $p \in F(p)$; the fixed point set of $F$ is the set of all fixed points of $F$ and is denoted by $\mathcal{F}(F)$. For a compactum $Z$, we use the phrase multi-valued fixed point set of $Z$ to refer to a subset, $A$, of $Z$ such that $A$ is the fixed point set of some multi-valued map $F: Z \rightarrow 2^{Z}$. In case that $F$ is continuum-valued, we call $A$ a continuum-valued fixed point set of $Z$.

The notion that was studied in [4] is defined as follows. A multi-valued absolute fixed point set (MAFS) is a compactum, $A$, such that whenever $A$ is embedded as a subspace, $A^{\prime}$, of any compactum, $Z$, then $A^{\prime}$ is a continuum-valued fixed point set of $Z$. It was shown that a compactum $A$ is a MAFS if and only if $A$ contains only finitely many components, all but at most one of which are locally connected $[4,1.1]$.

Received by the editors January 9, 1997 and, in revised form, April 17, 1997.

1991 Mathematics Subject Classification. Primary 54F15, 54C60; Secondary 54C15, 54B20, $54 \mathrm{D} 05$.

Key words and phrases. Compactum, continuum, hyperspace, locally connected, multi-valued absolute fixed point set, MAFS, multi-valued map.

(C) 1998 American Mathematical Society 
Let $Z$ be a compactum, and let $n \in\{1,2, \cdots, \omega\}$ where $\omega$ denotes the first infinite cardinal number. We define $C_{n}(Z)$ for each $n$ as follows:

$$
C_{n}(Z)=\left\{A \in 2^{Z}: A \text { has at most } n \text { components }\right\} .
$$

In particular, if $A \in C_{\omega}(Z)$, then $A$ has at most countably many components.

The notion that we study in this paper may now be defined as follows. An $n$ MAFS is a compactum, $A$, such that whenever $A$ is embedded as a subspace, $A^{\prime}$, of any compactum, $Z$, then there is some map $F: Z \rightarrow C_{n}(Z)$ for which $\mathcal{F}(F)=A^{\prime}$.

We now state the characterizations that we will prove.

1.1 Theorem. Let $A$ be a compactum, and let $n \in\{1,2, \ldots\}$. Then, $A$ is an $n$ MAFS if and only if $A$ has only finitely many components, all but at most $n$ of which are locally connected.

1.2 Theorem. Let $A$ be a compactum. Then, $A$ is an $\omega-M A F S$ if and only if $A$ has at most countably many components.

We remark that the notions of MAFS and 1-MAFS are identical, and that the characterization given in 1.1 for every finite $n$ is analogous to that given for the class of MAFS. Therefore, it seems natural to consider an inductive argument for 1.1. However, such a proof seems to be elusive.

\section{Proof of Theorem 1.1}

The following lemmas will be used several times throughout this paper; the proof of 2.1 is similar that given for 1.43 in [5] and will be omitted.

2.1 Lemma. Let $Z$ be a compactum and let $\Lambda \subseteq 2^{Z}$. If $\Lambda$ is connected and $\Lambda \cap$ $C_{n}(Z) \neq \varnothing$, then $\bigcup \Lambda \in C_{n}(Z)$, where $n \in\{1,2, \cdots, \omega\}$.

The proof of the following lemma is similar to that given for 3.1 in [4] and will be omitted.

2.2 Lemma. Every retract of an $n$-MAFS is an $n$-MAFS for $n \in\{1,2, \cdots, \omega\}$.

The result in 2.3 will be used in 2.4 to provide a necessary condition for a compactum to be an $n$-MAFS for any finite $n$. A second necessary condition will be given in 2.5 .

2.3 Proposition. A simple sequence is not an $n$-MAFS for any $n \in\{1,2, \cdots\}$.

Proof. Let $J=\left\{(0,0, z) \in \mathbf{R}^{3}: 0 \leq z \leq 2\right\}$ and $J^{\prime}=\left\{(0,0, z) \in \mathbf{R}^{3}: 0 \leq z \leq 1\right\}$. Define $W_{0}=\left\{\left(x, 0,1+\sin \frac{1}{x}\right): 0<x \leq 1\right\}$. For each $i=1,2, \cdots$, define $W_{i}$ to be a $\sin \frac{1}{x}$ curve in $\mathbf{R}^{3}$ whose height near the $z$-axis is half its height elsewhere, and arrange these curves around the $z$-axis in such a way that they converge to $W_{0}$. Specifically, define $W_{i}$ to be an isometric copy of

$$
\left\{\left(x, \frac{1}{2}\left(1+\sin \frac{1}{x}\right)\right): 0<x \leq \frac{2}{(4 i+3) \pi}\right\} \cup\left\{\left(x, 1+\sin \frac{1}{x}\right): \frac{2}{(4 i+3) \pi} \leq x \leq 1\right\}
$$

in $\mathbf{R}^{3}$ such that

(i) $W_{i} \cap W_{j}=\varnothing$ whenever $i \neq j(i, j \in\{0,1,2, \cdots\})$,

(ii) $\overline{W_{i}}-W_{i}=J^{\prime}(i \in\{1,2, \cdots\})$,

(iii) $\lim W_{i}=W_{0}$. 
Define $Z=J \cup \bigcup_{i=0}^{\infty} W_{i}$. For convenience, we will refer to a point $(0,0, a)$ as $a$, and we will use interval notation in the natural way to denote connected subsets of $J$; in particular, 2 denotes $(0,0,2)$, and $[0,2]=J$.

Let $p_{i}$ denote the point of $W_{i}$ that corresponds to $\frac{2}{(4 i-1) \pi}$ for each $i=1,2, \cdots$; observe that there is a subset of $W_{i}$ with height 2 between $p_{i}$ and the $z$-axis. Set $A=\{0\} \cup\left\{p_{1}, p_{2}, \cdots\right\}$. Then $A$ is a simple sequence in $Z$ with limit point 0 . Let $F: Z \rightarrow C_{n}(Z)$ be any map from $Z$ to $C_{n}(Z)$. We will show that $A$ cannot be the fixed point set of $F$.

For the purpose of contradiction, suppose that $\mathcal{F}(F)=A$. Observe that if there was some $x \in(0,2]$ for which some component of $F(x)$ was contained above $x$ in $J$, then the continuity of $F$ would imply that some point above $x$ is fixed by $F$. However, this is impossible by the assumption that $\mathcal{F}(F) \cap J=\{0\}$. It follows that

(1) no component of $F(x)$ lies above $x$ in $J$ for all $x \in(0,2]$.

Now, suppose that

$$
J \subseteq F(0)
$$

Then, by the continuity of $F$, there is some $\epsilon \in(0,1)$ for which

(2) some subset of $F(x)$ lies near $J$ for each $x \in[0, \epsilon]$.

Note also that since $(0, \epsilon) \cap A=\varnothing$, we have that

(3) $J^{\prime} \nsubseteq F(x)$ for any $x \in(0, \epsilon)$.

Choose any $x \in(0, \epsilon)$. By (1) and (2), at least one component of $F(x)$ lies in $\bigcup_{i=0}^{\infty} W_{i}$. Also, since $F(x) \in C_{n}(Z)$, (3) gives that there are only $k \leq n$ many members of $\left\{W_{i}: i=0,1,2, \cdots\right\}$ which meet $F(x)$, say $W_{i_{1}}, W_{i_{2}}, \cdots, W_{i_{k}}$. Let $y$ be any other member of $(0, \epsilon)$. Using the continuity of $F$, we see that if $F(y) \cap W_{j} \neq \varnothing$ for some $j \notin\left\{i_{1}, \cdots, i_{k}\right\}$, then $J^{\prime} \subseteq F(z)$ for some $z \in[x, y]$, contradicting (3). It follows that $F(x) \subseteq J^{\prime} \cup W_{i_{1}} \cup \cdots \cup W_{i_{k}}$ for every $x \in(0, \epsilon)$. Therefore, we have by continuity that $F(0) \subseteq J^{\prime} \cup \overline{W_{i_{1}}} \cup \cdots \cup \overline{W_{i_{k}}}$. So, from $(*)$ we conclude that $i_{j}=0$ for some $j \in\{1, \cdots, k\}$. Thus, (3) gives that for each $x \in(0, \epsilon)$, some component of $F(x)$ is properly contained in $W_{0}$. It follows from the continuity of $F$ that there is some $x_{0} \in W_{0}$ that lies between $J$ and some component of $F\left(x_{0}\right)$ in $W_{0}$; we conclude that $W_{0}$ contains a fixed point of $F$, contrary to our supposition that $\mathcal{F}(F)=A$. Therefore, $(*)$ cannot occur.

Suppose on the other hand that

$$
J \nsubseteq F(0) .
$$

Observe that if $2 \in F(0)$, then $(* *)$ implies that some component of $F(0)$ lies strictly above 0 in $J$; however, it would then follow by continuity that some component of $F(x)$ lies strictly above $x$ for some $x>0$. Since this would contradict (1), we must have that $2 \notin F(x)$ for every $x \in J$. Therefore, the continuity of $F$ gives that each $x \in J$ is contained in an open subset, $U_{x}$, of $Z$ for which $2 \notin F(y)$ for every $y \in U_{x}$. Thus, $U=\bigcup_{x \in J} U_{x}$ is an open subset of $Z$ containing $J$ for which

(4) $2 \notin F(y)$ for every $y \in U$.

Observe that $U$ contains a subcontinuum, $Y$, such that $J \subseteq Y$ and $p_{i} \in Y$ for all $i$ sufficiently large. Since $F$ is continuous, we have that $F(Y)$ is connected; thus, $\bigcup F(Y)$ belongs to $C_{n}(Z)$ by 2.1. Furthermore, our assumption that $\mathcal{F}(F)=A$ implies that $\bigcup F(Y)$ contains $p_{i}$ for $i$ sufficiently large. Thus, since $\bigcup F(Y)$ contains at most $n<\infty$ components, some component, $K$, of $\bigcup F(Y)$ must contain $p_{i}$ for 
infinitely many $i$. Since $K$ is a closed connected subset of $\bigcup F(Y)$ that contains infinitely many members of $\left\{p_{i}: i=1,2, \cdots\right\}$, it follows from our choice of $p_{i}$ that $[0,2] \subseteq K$, contrary to $(4)$. Hence, $(* *)$ cannot occur. Since we can have neither $(*)$ nor $(* *)$, we must conclude that our supposition that $\mathcal{F}(F)=A$ is false. So, $A$ cannot be the fixed point set of any $F: Z \rightarrow C_{n}(Z)$. This proves 2.3.

2.4 Corollary. Every $n$-MAFS contains at most finitely many components for each $n \in\{1,2, \cdots\}$.

Proof. Let $n \in\{1,2, \cdots\}$. If $A$ is a compactum with infinitely many components, then $A$ contains a simple sequence as a retract $[4,3.2]$. It follows immediately from 2.2 and 2.3 that $A$ is not an $n$-MAFS.

2.5 Proposition. Let $n \in\{1,2, \cdots\}$. If $A$ is an $n$-MAFS, then all but at most $n$ components of A must be locally connected.

Proof. By 2.2 and 2.4, it suffices to show that

(*) if $A$ is the disjoint union of $n+1$ nonlocally connected continua, then $A$ is not an $n$-MAFS.

Let $A_{1}, \cdots, A_{n+1}$ denote the components of $A$. Since $A_{1}$ is not locally connected at some $a_{\infty} \in A_{1}$, there exists a neighborhood, $V$, of $a_{\infty}$, and points $a_{i} \in V$, $i=1,2, \cdots$, for which $a_{\infty}=\lim a_{i}$ and such that

(1) no two of $a_{\infty}, a_{1}, a_{2}, \cdots$ lie in the same component of $V$,

(2) $C_{\infty}=\lim C_{i}$, where $C_{i}$ denotes the component of $a_{i}$ in $V$ for each $i \in$ $\{1,2, \cdots, \infty\}$.

Let $S=\left\{\left(x, \sin \frac{1}{x}\right): 0<x \leq 1\right\}$. For each $i \in\{1,2, \cdots\}$ and each $j \in\{1,2, \cdots, i\}$, let $f_{i j}: S \rightarrow \mathbf{R}^{3}$ be an embedding for which

(3) $\overline{f_{i j}(S)}-f_{i j}(S)=\{0\} \times\{0\} \times\left[0, \frac{1}{j}\right]=I_{j}$,

(4) $f_{i j}(S) \subset\left\{(x, y): x^{2}+y^{2} \leq \frac{1}{k}\right\} \times I_{j}$, where $k=\frac{i(i-1)}{2}+j$,

(5) $f_{i j}(S) \cap f_{i^{\prime} j^{\prime}}(S) \neq \varnothing$ if and only if $i=i^{\prime}$ and $j=j^{\prime}$.

In other words, $\left\{f_{i j}(S)\right\}$ is a sequence of $\sin \frac{1}{x}$ curves with decreasing widths and alternating heights. The first term, $f_{11}(S)$, fits inside the tube centered on the $z$-axis with radius 1 , while the fifth term, $f_{32}(S)\left(5=\frac{3(3-1)}{2}+2\right)$, fits inside the tube centered on the $z$-axis with radius $\frac{1}{5}$; also, $f_{11}(S)$ has height 1 , and the heights of $f_{31}(S), f_{32}(S)$, and $f_{33}(S)$ have heights $1, \frac{1}{2}$, and $\frac{1}{3}$ respectively. Define $p=(0,0,0)$ and $q_{j}=\left(0,0, \frac{1}{j}\right)$ for each $j \in\{1,2, \cdots\}$. For each $i \in\{1,2, \cdots\}$ and each $j \in\{1, \cdots, i\}$, let $p_{i j}=p_{k}$ be a point of $f_{i j}(S)$ such that $p=\lim p_{i}$, where $k=\frac{i(i-1)}{2}+j$. Define $Y_{1}=\bigcup_{\substack{i<\infty \\ j \leq i}} \overline{f_{i j}(S)}$. Without loss of generality, we may assume that $A_{1}$ and $Y_{1}$ are disjoint. Let $h_{i}: Y_{1} \rightarrow \mathbf{R}^{3}$ be an embedding for each $i=1,2, \cdots, n+1$ such that $h_{1}\left(Y_{1}\right)=Y_{1}, h_{i}\left(Y_{1}\right) \cap h_{j}\left(Y_{1}\right)=\varnothing$ whenever $i \neq j$, and $h_{i}\left(Y_{1}\right) \cap A=\varnothing$ for all $i=1,2, \cdots, n+1$. Set $Y_{i}=h_{i}\left(Y_{1}\right)$ for each $i=1,2, \cdots, n+1$. Form $Z_{1}$ from $A_{1}$ and $Y_{1}$ by identifying $a_{\infty}$ with $p$, and $a_{k}$ with $p_{k}$ for each $k=1,2, \cdots$ and define $Z_{i}$ from $A_{i}$ and $Y_{i}$ in a similar manner. Let $T$ be a simple $(n+1)$-od $[6,9.8]$ with vertex $t$ and endpoints $t_{1}, \cdots t_{n+1}$ such that $T \cap \bigcup_{i=1}^{n+1} Z_{i}=\varnothing$. Define $Z$ to be the continuum obtained by identifying each $t_{i}$ with the image of $h_{i}(p) \in Z_{i}$. Then $Z$ is a continuum that contains a homeomorphic copy of $A$. We will use $A, V, C_{i}, I_{i}, Y_{i}$, and $T$ to denote the natural copies of these spaces in $Z$. 
Suppose that there is some map $F: Z \rightarrow C_{n}(Z)$ with $\mathcal{F}(F)=A$. Note that since $F(t)$ has at most $n$ components, we may assume, without loss of generality, that $F(t)$ shares no point in common with the component of $Z_{1}$ in $Z-\{t\}$. It follows from the continuity of $F$, and the supposition that $\mathcal{F}(F)=A$, that

(6) $F(p) \cap Z_{1}=\{p\}$.

By (6) and the continuity of $F$, there is some open set $U$ of $Z$ with $p \in U$ for which

(7) $Z_{1} \cap F(x) \subseteq V \cup Y_{1}$ for each $x \in U$.

It is clear from (3) that $U$ contains $I_{j_{0}}$ for some $j_{0} \in\{1,2, \cdots\}$; indeed, it is clear from (4) that $U$ contains $\overline{f_{i j_{0}}(S)}$ for all sufficiently large $i$. Note that from (6), the continuity of $F$, and the supposition that $\mathcal{F}(F)=A$, we have that $q_{j_{0}} \notin F(x)$ for any $x \in I_{j_{0}}$. Thus, for each $x \in I_{j_{0}}$, there is an open set, $V_{x} \subseteq U$, such that $q_{j_{0}} \notin F(y)$ for every $y \in V_{x}$. So, $U^{\prime}=\bigcup_{x \in I_{j_{0}}} V_{x}$ is an open subset of $U$ for which

(8) $q_{j_{0}} \notin F(y)$ for every $y \in U^{\prime}$.

Let $E$ be a subcontinuum of $U^{\prime}$ that contains $p_{i j_{0}}$ for all sufficiently large $i$. Then $F(E)$ is a subcontinuum of $C_{n}(Z)$, and so $\bigcup F(E)$ belongs to $C_{n}(Z)$ by 2.1. Since each $p_{i j_{0}}$ belongs to $\mathcal{F}(F)$, it follows that some component, $K$, of $\bigcup F(E)$ must contain infinitely many members of $\left\{p_{i j_{0}}: i=1,2, \cdots\right\}$. Hence, $K$ must also contain $p$. Observe that $K \subseteq V \cup Y_{1}$ by (7). So by (1), $\overline{K-V}$ is a subcontinuum of $Y_{1}$ that contains $p$ and infinitely many members of $\left\{p_{i j_{0}}: i=1,2, \cdots\right\}$. But, by (5), any subcontinuum of $Y_{1}$ that contains both $p$ and some $p_{i j_{0}}$ must also contain $I_{j_{0}}$. Thus, $q_{j_{0}} \in K$, contrary to (8). So, we must conclude that our supposition that $\mathcal{F}(F)=A$ is false. This shows $(*)$, as required. Thus, we have proven 2.5.

The following proposition asserts that the properties described in 2.4 and 2.5 are, together, sufficient for a compactum to be an $n$-MAFS.

2.6 Proposition. Let $Z$ be a compactum, and let $n \in\{1,2, \cdots\}$. If $A$ is a compactum in $Z$ that contains only finitely many components, all but at most $n$ of which are locally connected, then $A=\mathcal{F}(F)$ for some map $F: Z \rightarrow C_{n}(Z)$.

Proof. Let $A=A_{1} \cup A_{2} \cup \cdots \cup A_{m}$, where $A_{i}$ is a component of $A$ for each $i \in$ $\{1, \cdots, m\}$. Note that if $m \leq n$, then $F: Z \rightarrow C_{n}(Z)$, given by $F(z)=A$ for every $x \in Z$, is a map with $\mathcal{F}(F)=A$. So we assume that $m>n$. Without loss of generality, we also assume that $A_{n+1}, \cdots, A_{m}$ are locally connected. It follows from the characterization of the class of MAFS $[4,1.1]$ that there is a map $G: Z \rightarrow C(Z)$ with $\mathcal{F}(G)=A_{n} \cup A_{n+1} \cup \cdots \cup A_{m}$. Define $F: Z \rightarrow C_{n}(Z)$ by

$$
F(x)=G(x) \cup A_{1} \cup \cdots \cup A_{n-1}
$$

for each $x \in Z$. Then $F$ is a map with $\mathcal{F}(F)=A$. This proves 2.6.

The theorem in 1.1 has now been proved by 2.4, 2.5, and 2.6.

\section{Proof of Theorem 1.2}

3.1 Proposition. Every compactum with at most countably many components is an $\omega-M A F S$.

Proof. Let $Z$ be a compactum, and let $A$ be a compact subset of $Z$ with at most countably many components. Then $F: Z \rightarrow C_{\omega}(Z)$ defined by $F(x)=A$ for all $x \in Z$ is a map with $\mathcal{F}(F)=A$. This proves 3.1. 
The rest of the work in this section is directed toward demonstrating that having only countably many components is also a necessary condition for a compactum to be an $\omega$-MAFS.

3.2 Lemma. An $\omega$-MAFS cannot be both totally disconnected and uncountable.

Proof. Let $A$ be an uncountable, totally disconnected compactum. Without loss of generality, we may assume that $A$ is a subset of the circle in $\mathbf{R}^{3}$ given by

$$
\left\{(x, y, 1+\sin 1): x^{2}+y^{2}=1\right\} .
$$

Let $U_{1} \supset U_{2} \supset \cdots$ be a strictly decreasing sequence of uncountable, simultaneously open and closed subsets of $A$ such that $U_{1}=A$ and $\bigcap_{i=1}^{\infty} U_{i}=\{p\}$ for some $p \in A$. Define $J, J^{\prime}$ and $W_{i}(i \in\{1,2, \cdots\})$ as we did in the proof of 2.3. Now, for each $a \in A-\{p\}$, observe that there is precisely one $i_{a} \in\{1,2, \cdots\}$ for which $a \in U_{i_{a}}-U_{i_{a}+1}$; let $h_{a}: W_{i_{a}} \rightarrow \mathbf{R}^{3}$ be the isometric embedding for which

$$
\begin{gathered}
h_{a}((1,1+\sin 1))=a, \\
\overline{h_{a}\left(W_{i_{a}}\right)}-h_{a}\left(W_{i_{a}}\right)=J^{\prime} .
\end{gathered}
$$

Let $S=\left\{\left(x, 1+\sin \frac{1}{x}: 0<x \leq 1\right\}\right.$, and let $h_{p}: S \rightarrow \mathbf{R}^{3}$ be the isometric embedding for which

$$
\begin{gathered}
h_{p}((1,1+\sin 1))=p, \\
\overline{h_{p}(S)}-h_{p}(S)=J
\end{gathered}
$$

Let $Z=\overline{h_{p}(S)} \cup \bigcup_{a \in A-\{p\}} h_{a}\left(W_{i_{a}}\right)$. Now, for each $a \in A$, define

$$
x_{a}= \begin{cases}h_{a}\left(\frac{2}{\left(4 i_{a}-1\right) \pi}\right), & \text { if } a \neq p, \\ (0,0,0), & \text { if } a=p .\end{cases}
$$

Observe that the map given by $a \mapsto x_{a}$ is an embedding of $A$ into $Z$. Set $A^{\prime}=$ $\left\{x_{a}: a \in A\right\}$, and suppose that there exists some $F: Z \rightarrow C_{\omega}(Z)$ with $\mathcal{F}(F)=A^{\prime}$. This supposition leads to a contradiction by an argument similar to the one given for 2.3. This proves 3.2.

The following technical lemma will facilitate the proof of 3.4.

3.3 Lemma. Let $Z$ be a compactum, and let $V$ be an open subset of $Z$ which meets uncountably many components of $Z$. Then, for each $n \in\{1,2, \cdots\}$ there exist points $p_{1}, \cdots, p_{n}$ of $V$ which lie in $n$ distinct components of $Z$, and for which any open set containing $p_{i}$ meets uncountably many components of $Z$ for each $i=1, \cdots, n$.

Proof. Suppose that the conclusion fails for $n=1$; that is, suppose that

(*) for every $p \in V$, there is some open subset of $V$ that contains $p$ and meets at * most countably many components of $Z$.

Let $\mathcal{C}$ denote the collection of all components of $Z$ that meet $V$. For each $C \in \mathcal{C}$, let $z_{C} \in C \cap V$; define $A=\left\{z_{C}: C \in \mathcal{C}\right\}$. Then, by hypothesis, we have that

(1) $A$ is uncountable.

Now, let $\left\{V_{i}\right\}_{i=1}^{\infty}$ be a sequence of open subsets of $V$ for which $c l_{Z}\left(V_{i}\right) \subseteq V$ and for which $\bigcup_{i=1}^{\infty} V_{i}=V$. Clearly we have that

(2) $\bigcup_{i=1}^{\infty}\left(c l_{Z}\left(V_{i}\right) \cap A\right)=A$. 
Therefore, since the union of countably many countable sets is countable, it follows immediately from (1) and (2) that $\operatorname{cl}_{Z}\left(V_{k}\right) \cap A$ is uncountable for some $k \in$ $\{1,2, \cdots\}$. Setting $E=c_{Z}\left(V_{k}\right)$ shows that

(3) $E$ is a closed subset of $Z$ in $V$ that meets uncountably many components of $Z$.

By $(*)$ and the compactness of $E$, there exist finitely many open subsets, $U_{1}, \cdots, U_{m}$, of $V$ for which $E \subseteq \bigcup_{i=1}^{m} U_{i}$ and such that each $U_{i}$ meets at most countably many components of $Z$. But this implies that $E$ meets at most countably many components of $Z$, contrary to $(3)$. Hence, the supposition in $(*)$ is false. Therefore, the lemma holds for $n=1$.

Now suppose that the lemma holds for $n=j$. Let $p_{1}, \cdots, p_{j}$ be points of $V$ that satisfy the conclusion of the lemma, and let $K_{i}$ denote the component of $p_{i}$ in $Z$ for each $i \in\{1, \cdots, j\}$. Set $K=\bigcup_{i=1}^{j} K_{i}$. Then $V-K$ is an open subset of $Z$ that meets uncountably many components of $Z$. So, by the case for $n=1$, there is some point $p_{j+1} \in V-K$ for which any open set containing $p_{j+1}$ meets uncountably many components of $Z$. This completes the proof of 3.3 .

The following notation will facilitate the proof of our next result. Let $\{0,1\}<\omega$ denote the set of all finite sequences with range in $\{0,1\}$, that is,

$$
\{0,1\}^{<\omega}=\left\{\left\langle a_{1}, \cdots, a_{n}\right\rangle: n=1,2, \cdots ; a_{i}=0,1 ; \quad i=1, \cdots, n\right\} \cup\{\varnothing\} .
$$

For any $s \in\{0,1\}^{<\omega}$, let $|s|$ denote the length of $s$. Let $s 0$ and $s 1$ denote those elements of $\{0,1\}^{<\omega}$ that have length $|s|+1$, that agree with $s$ in the first $|s|$ positions, and that have 0 and 1 , respectively, in the final position. If $|s|=1$, set $s^{-}=\varnothing$; otherwise, let $s^{-}$denote that element of $\{0,1\}^{<\omega}$ of length $|s|-1$ that agrees with $s$ in the first $|s|-1$ positions. Finally, let $\{0,1\}^{\omega}$ denote the set of all countably infinite sequences with range in $\{0,1\}$.

3.4 Lemma. Let $Z$ be a compactum with uncountably many components. Then $Z$ has a retract which is both uncountable and totally disconnected.

Proof. Using 3.3, there exist distinct components, $K_{\langle 0\rangle}$ and $K_{\langle 1\rangle}$, of $Z$, such that for some $p_{\langle i\rangle} \in K_{\langle i\rangle}$, every open set about $p_{\langle i\rangle}$ meets uncountable many components of $Z$. Using the fact that every component of $Z$ is also a quasicomponent of $Z$ [ 6 , 5.18], let $U_{\langle i\rangle}$ be a simultaneously closed and open (clopen) subset of $Z$ for each $i=0,1$ such that $K_{\langle i\rangle} \subseteq U_{\langle i\rangle}, U_{\langle 0\rangle} \cap U_{\langle 1\rangle}=\varnothing$, and $U_{\langle 0\rangle} \cup U_{\langle 1\rangle} \neq Z$. Choose any point, $p_{\varnothing}$, in $Z-\left(U_{\langle 0\rangle} \cup U_{\langle 1\rangle}\right)$, and define $f_{\varnothing}: Z-\left(U_{\langle 0\rangle} \cup U_{\langle 1\rangle}\right) \rightarrow Z$ by

$$
f_{\varnothing}(x)=p_{\varnothing} \text { for all } x \in Z-\left(U_{\langle 0\rangle} \cup U_{\langle 1\rangle}\right) \text {. }
$$

We will now construct $U_{s}, V_{s}, K_{s}, p_{s}$, and $f_{s}$ for each $s \in\{0,1\}<\omega$ by induction on $\{0,1\}^{<\omega}$. Set $V_{\varnothing}=U_{\varnothing}=Z$, and let $s \in\{0,1\}<\omega$. Let $V_{s}$ be an open set about $p_{s}$ such that

(1) the diameter of $V_{s}$ is $\leq 2^{-|s|}$,

(2) $V_{s} \subseteq U_{s} \cap V_{s^{-}}$.

By applying 3.3 to $U_{s}$, we can find distinct components, $K_{s 0}$ and $K_{s 1}$, of $U_{s}$ (hence, of $Z$ ), for which

(3) $K_{s i} \neq K_{s}$ for $i=0,1$,

(4) there is some $p_{s i} \in K_{s i} \cap V_{s}$ such that every open set about $p_{s i}$ meets uncountably many components of $Z$. 
Let $U_{s i}$ be a clopen subset of $Z$ for each $i=0,1$ such that

(5) $U_{s i} \subseteq U_{s}$,

(6) $K_{s i} \subseteq U_{s i}$,

(7) $U_{s 0} \cap U_{s 1}=\varnothing$,

(8) $U_{s i} \cap K_{s}=\varnothing$.

Then, define $f_{s}: U_{s}-\left(U_{s 0} \cup U_{s 1}\right) \rightarrow Z$ by

$$
f_{s}(x)=p_{s} \text { for all } x \in U_{s}-\left(U_{s 0} \cup U_{s 1}\right) .
$$

Observe that since each $U_{s i}$ is open, (4) and (6) give that $U_{s i}$ contains uncountably many components of $Z$ for each $i=0,1$. This completes our construction. Now, for each $r \in\{0,1\}^{\omega}$, let $r_{n}$ denote the unique element of $\{0,1\}^{<\omega}$ of length $n$ for which $r_{n}(k)=r(k)$ for all $k \in\{1, \cdots, n\}$. Let $Z^{\prime}=\bigcup\left\{\bigcap_{n=1}^{\infty} U_{r_{n}}: r \in\{0,1\}^{\omega}\right\}$. It is easy to verify that

(9) $x \in Z-Z^{\prime}$ if and only if $x \in U_{s}-\left(U_{s 0} \cup U_{s 1}\right)$ for some $s \in\{0,1\}^{<\omega}$.

Since $U_{s}-\left(U_{s 0} \cup U_{s 1}\right)$ is open for every $s \in\{0,1\}^{<\omega}$, (9) gives that

(10) $Z^{\prime}$ is a closed subset of $Z$.

Define $f^{\prime}: Z-Z^{\prime} \rightarrow Z$ by

$$
f^{\prime}(x)=f_{s}(x) \text { if } x \in U_{s}-\left(U_{s 0} \cup U_{s 1}\right), \quad s \in\{0,1\}^{<\omega} .
$$

Note that $f^{\prime}$ is well defined by (5), (7), and (9). Furthermore, since $f_{s}$ is constant for each $s \in\{0,1\}^{<\omega}$ and each $U_{s}-\left(U_{s 0} \cup U_{s 1}\right)$ is open in $Z$, it is clear that

(11) $f^{\prime}$ is continuous.

For each $r \in\{0,1\}^{\omega}$, use (1) and (2) to define $p_{r}=\bigcap_{n=1}^{\infty} \overline{V_{r_{n}}}$. Then, define $f: Z \rightarrow Z$ by

$$
f(x)=\left\{\begin{array}{ll}
f^{\prime}(x) & \text { if } x \in Z-Z^{\prime}, \\
p_{r}, & \text { if } x \in \bigcap_{n=1}^{\infty} U_{r_{n}}
\end{array} \text { for some } n .\right.
$$

Again, $f$ is well defined by (5), (7), and (9).

By (10) and (11), we have that $f$ is continuous at each point of $Z-Z^{\prime}$. So, to see that $f$ is continuous on $Z$, it is enough to show that $f$ is continuous at each $x \in Z^{\prime}$. Let $x_{0} \in Z^{\prime}$, and let $\left\{x_{i}\right\}_{i=1}^{\infty}$ be a sequence in $Z$ with $\lim x_{i}=x_{0}$. By the definitions of $Z^{\prime}$ and $f$, there is some $r \in\{0,1\}^{\omega}$ for which $f\left(x_{0}\right)=p_{r}$. Let $V$ be an open subset of $Z$ about $f\left(z_{0}\right)=p_{r}$. Then, by (1), (2), and the definition of $p_{r}, V$ contains $V_{r_{N}}$ for some $N \in\{1,2, \cdots\}$. By the definition of $f$, we have that $x_{0} \in U_{r_{N+1}}$; hence, $x_{i} \in U_{r_{N+1}}$ for all $i$ sufficiently large. But, by (2), (4), and the definitions of $f_{s}, p_{r}$, and $f$, it follows that $f\left(U_{r_{N+1}}\right) \subseteq V_{r_{N}}$. So $f\left(x_{i}\right) \in V$ for all $i$ sufficiently large; therefore, $f$ is continuous at $x_{0}$, as required.

Finally, observe that by (2), (3), (4), and (8), $f(Z) \cap K$ contains at most one point from each component, $K$, of $Z$; hence, we have that $f(Z)$ is totally disconnected. Furthermore, it is clear from our construction that $f(Z)$ is a retract of $Z$ with uncountably many elements. This proves 3.4.

3.5 Proposition. Every $\omega-M A F S$ contains at most countably many components.

Proof. This follows immediately from 2.2, 3.2 and 3.4.

The theorem in 1.2 has now been proved by 3.1 and 3.5 . 
3.6 Remark. Let $\mathfrak{c}$ denote the cardinality of $\mathbf{R}$. By defining a $\mathfrak{c}$-MAFS in the natural way, it is easily seen that every compactum is a $\mathfrak{c}$-MAFS since every compactum has at most $\mathfrak{c}$ components.

3.7 Problem. Characterize the class of $n$-MAFS, $n \in\{1,2, \cdots, \omega\}$, in the setting of compact Hausdorff spaces.

\section{ACKNOWLEDGMENTS}

The results in this paper are a part of the author's doctoral dissertation, Absolute Fixed Point Sets for Multi-Valued Maps, completed at West Virginia University under the direction of Sam B. Nadler, Jr. The author wishes to thank Professor Nadler for his enthusiastic support and assistance.

\section{REFERENCES}

1. John R. Martin, On absolute fixed-point sets, Colloq. Math. 35 (1976), 67-71. MR 53:4033

2. _ _ Absolute fixed-point sets in compacta, Colloq. Math. 39 (1977), 41-44. MR 80f:54028

3. $\quad$ Absolute fixed point sets and AR-spaces, Fund. Math. 112 (1981), 159-164. MR 82j:54098

4. Eric L. McDowell and Sam B. Nadler, Jr., Absolute fixed point sets for continuum-valued maps, Proc. Amer. Math. Soc. 124 (1996), 1271-1276. MR 96g:54042

5. Sam B. Nadler, Jr., Hyperspaces of sets, Marcel Dekker Inc., New York, 1978. MR 58:18330

6. Continuum theory, an introduction, Marcel Dekker, Inc., New York, 1992. MR 93m:54002

Department of Mathematics, Bethany College, Bethany, West Virginia 26032

E-mail address: e.mcdowell@mail.bethanywv.edu

Current address: Department of Mathematical Sciences, Berry College, Mount Berry, Georgia 30149

E-mail address: emcdowell@berry.edu 